\title{
Intraoperative specimen analysis in prostate cancer surgery using Cerenkov Luminescence Imaging
}

\author{
J. olde Heuvel*a,b B. J. de Wit-van der Veen ${ }^{\mathrm{a}}$, H.G. van der Poel ${ }^{\mathrm{c}}$, M.P.M. Stokkel ${ }^{\mathrm{a}}$, C.H. Slump ${ }^{\mathrm{b}}$ \\ ${ }^{a}$ Department of Nuclear Medicine, Netherlands Cancer Institute-Antoni van Leeuwenhoek, \\ Amsterdam, The Netherlands; ${ }^{\mathrm{b}}$ Robotics and Mechatronics Group, Technical Medical Centre, \\ University of Twente, Enschede, The Netherlands; ${ }^{\circ}$ Department of Urology, Netherlands Cancer \\ Institute-Antoni van Leeuwenhoek, Amsterdam, The Netherlands; \\ *Contact: j.olde.heuvel@nki.nl
}

\begin{abstract}
Intraoperative margin assessment during prostate cancer $(\mathrm{PCa})$ surgery might reduce the number of positive surgical margins (PSM). Cerenkov Luminescence Imaging (CLI) based on optical imaging of PET radiopharmaceuticals is suitable for this purpose. Previous CLI research has been conducted with ${ }^{18}$ Fluorine, however, ${ }^{68}$ Gallium has more favorable CLI properties and can be coupled to a prostate cancer specific tracer: the prostate-specific membrane antigen $\left({ }^{68} \mathrm{Ga}\right.$-PSMA). Light yield, resolution and camera sensitivity of ${ }^{68} \mathrm{Ga}$ and ${ }^{18} \mathrm{~F}$ for CLI were investigated in a pre-clinical setting. CLI images were acquired using the LightPath system, with an exposure time of 120 s, $2 \times 2$ binning and $300 \mathrm{~s}, 8 \times 8$ binning. Three Eppendorf tubes $(1 \mathrm{~mL})$ with different radioactivity concentrations $(2.5,10$ and $40 \mathrm{kBq} / \mathrm{mL}$ ) of ${ }^{18} \mathrm{~F}$ and ${ }^{68} \mathrm{Ga}$ were imaged. For both isotopes, an excellent linear relationship between the radioactivity concentration and detected light yield was observed $\left(\mathrm{R}^{2}=0.99\right) .{ }^{68} \mathrm{Ga}$ showed $22 \times$ more light yield compared to ${ }^{18} \mathrm{~F}$, thus enabled lower detectable radioactivity concentration levels $(1.2 \mathrm{vs} .23 .7 \mathrm{kBq} / \mathrm{mL})$. Based on these promising results, a prospective feasibility study for intraoperative prostate cancer specimen CLI measurements with ${ }^{68} \mathrm{Ga}$-PSMA was designed and the first patients were enrolled in this study. The prostate was imaged ex vivo with the LightPath system $\sim 70$ minutes after injection of $\sim 100 \mathrm{MBq}{ }^{68} \mathrm{Ga}$-PSMA. Hotspots on the CLI images were marked for comparison with histopathology and corresponded to a PSM, defined as tumor on ink. In the first patients, CLI correctly identified all patients with a PSM. The encouraging preliminary results motivated for continuation of this trial.
\end{abstract}

Keywords: Cerenkov Luminescence Imaging, Intraoperative assessment, ${ }^{68}$ Ga-PSMA

\section{INTRODUCTION}

Currently, approximately 11-38\% of prostate cancer (PCa) resections are not radical enough resulting in a so-called Positive Surgical Margin (PSM). Incomplete removal of cancer during PCa surgery is associated with increased likelihood of disease recurrence and poor patient outcomes, so generally adjuvant therapy is indicated ${ }^{1}$. Complete surgical excision remains difficult, as the surgeon is unable to distinguish between cancerous and non-cancerous tissue during the surgery itself. Intraoperative margin assessment may guide surgeons to ensure complete removal of the tumor and, as such, Cerenkov Luminescence Imaging (CLI) is a promising novel technique.

CLI involves the imaging of Cerenkov photons emitted from standard clinical isotopes such as Fluorine-18 and Gallium-68. Cerenkov photons are emitted when a $\beta$-particle is traveling in a dielectric medium faster than the phase velocity of light in that medium ${ }^{2}$. Though this light has a continues spectrum, the signal that can be detected during surgery is affected due to absorption in tissue. In order to detect this faint light, a cooled, electron-multiplying charge-coupled device (emCCD) in a light-sealed area is used. In a pre-clinical setting, this technique is already applied to assess biochemical processes with sub-millimetric resolution ${ }^{3-5}$. This combination has potential in margin assessment, as specific radiotracers are developed to target cancerous tissue and Cerenkov radiation has a superficial penetration depth in tissue.

Grootendorst et al. demonstrated the usability of CLI for margin assessment in breast cancer using ${ }^{18} \mathrm{~F}$ Fluorodeoxyglucose $\left({ }^{18} \mathrm{~F}-\mathrm{FDG}\right){ }^{6}$. The applicability of ${ }^{18} \mathrm{~F}$-FDG in $\mathrm{PCa}$ is, however, limited, as these tumors generally have a low glucose metabolism. Yet, the prostate-specific membrane antigen receptor (PSMA) is a far

Optics and lonizing Radiation, edited by Brian W. Pogue, Proc. of SPIE Vol. 11224,

$1122407 \cdot$ ? 2020 SPIE $\cdot$ CCC code: $1605-7422 / 20 / \$ 21 \cdot$ doi: $10.1117 / 12.2542650$ 
more specific target for PCa. Consequently, various different PSMA-ligands are developed that are coupled to either ${ }^{18} \mathrm{~F}$ or ${ }^{68} \mathrm{Gallium}\left({ }^{68} \mathrm{Ga}\right)$. Nowadays, ${ }^{68} \mathrm{Ga}$-PSMA is a routinely used radiopharmaceutical for PET/CT staging of high grade primary and metastatic PCa in Europe ${ }^{7}$. It has the added benefit that ${ }^{68} \mathrm{Ga}$ theoretically offers a higher CLI signal compared to ${ }^{18} \mathrm{~F}$ due to the higher initial positron energy ${ }^{8}$. Hence, ${ }^{68} \mathrm{Ga}$-PSMA-CLI probably enables detection of lower radioactivity levels during post-surgical assessment of the prostate specimen.

The present study was initiated to evaluate the usability of ${ }^{68} \mathrm{Ga}$-PSMA-CLI for margin assessment during PCa surgery. Before initiation of any clinical procedures, the imaging characteristics of ${ }^{68} \mathrm{Ga}$ were compared to ${ }^{18} \mathrm{~F}$ in a series of experiments that also formed the basis for the clinical acquisition protocols. The requirements for a clinical CLI acquisition protocol include: 1) low dosage of radioactivity, ideally less than the clinical PET/CT dose of $150 \mathrm{MBq}, 2)$ acquisition within 30 minutes, and 3) clear distinction between tumor-positive and -negative areas.

\section{METHODS}

\subsection{Cerenkov Luminescence Imaging system}

The LightPath optical imaging system (Lightpoint Medical Ltd., Chesham, UK) was used for both the in vitro validation and clinical CLI acquisitions in this study. The system is equipped with a camera lens $(\mathrm{F} / 0.95,512 \times 512$ pixels) coupled through optics to a $-80{ }^{\circ} \mathrm{C}$ cooled electron multiplying charge coupled device (EMCCD; Andor iXon Ultra 897). The EMCCD is shielded with a tungsten plate and folded optics are used to reduce the number of gamma photons striking the EMCCD sensor. A standard optical camera (F/1.4 lens, $1600 \times 1200$ pixels) was used to acquire white-light reference images. The system is equipped with a light-tight imaging chamber to shield from ambient light.

\subsection{Gallium-68 validation experiments}

In vitro experiments were conducted using clinically relevant activity levels retrospectively acquired from 30 primary prostate tumors measured on ${ }^{68} \mathrm{Ga}$-PSMA PET/CT . This evaluation was approved by the Institutional Review Board (IRB) of the Netherlands Cancer Institute (registration number IRBd18144). These routine PET/CT acquisitions were performed on Gemini TF systems (Philips Healthcare, Best, the Netherlands) from base of skull to mid-thigh (Matrix: $512 \times 512$, voxel: $4 \times 4 \times 4 \mathrm{~mm}$ ). The CT-scan parameters were as follows: $120 \mathrm{kV}, 150 \mathrm{mAs}$, and slice thickness $1.0 \mathrm{~mm}$, with an increment of $1.0 \mathrm{~mm}$. Images were reconstructed using BLOB-OS-TF ${ }^{9}$ (3D ordered subset iterative Time of Flight reconstruction 3 iterations \& 33 subsets), including corrections for time-of-flight, decay, randoms, dead time, scatter and attenuation. OsiriX MD DICOM viewer (v.9.2, Pixmeo SARL, Bernex, Switzerland) was used for quantification. Average uptake $(\mathrm{Bq} / \mathrm{mL})$ in the prostate tumor was measured with a spherical volume of interests (VOI) of at least $1.5 \mathrm{~cm}$ to minimize the partial volume effect.

In the following validation experiments 2-Deoxy-2- $\left({ }^{18} \mathrm{~F}\right)$ Fluoroglucose (IBA Molecular, Louvain-La-Neuve, Belgium) and ${ }^{68} \mathrm{Ga}$ coupled to Glu-urea-Lys (Ahx)-HBED-CC (ABX GmbH, Radeberg, Germany) were used. Eppendorf tubes were filled with radioactivity diluted in different concentrations $(2.5,12.5$ and $45 \mathrm{kBq} / \mathrm{mL})$ in $1 \mathrm{~mL}$ water. Images were acquired every 20 minutes for 4 subsequent hours to assess the linearity of the CLI-system. These acquisitions were performed with 120s exposure times and $2 \times 2$ binning (E120B2), and 300s with $8 \times 8$ binning (E300B8). Imaging signals were also determined in a simulated clinical ex vivo situation using $1 \mathrm{~mm}$ of overlying chicken breast tissue on the Eppendorf tubes. Finally, the spatial resolution was evaluated using a $1.1 \mathrm{~mm}$ outer diameter glass capillary filled with $33 \mathrm{kBq}$ of ${ }^{18} \mathrm{~F}$ and ${ }^{68} \mathrm{Ga}$. The Full Width Half Maximum (FWHM) was determined on raw imaging data without filtering, with background correction and normalized for differences in activity levels between both isotopes. This experiment was repeated with the addition of chicken breast tissue. For more details we refer to ${ }^{10}$.

The acquired CLI images were processed with LightPath software, using a median filter ( $3 \times 3$ pixels) and Gaussian filter ( $\sigma=3$ pixels) to reduce the noise from high energy photons, also known as gamma strikes. The radiance level (photons $/ \mathrm{s} / \mathrm{cm}^{2} / \mathrm{sr}$ ) was used to compare the signal of both radionuclides. The average radiance was obtained by manual selection of a region of interest (ROI) around the Cerenkov signal on the EMCCD image of the tubes, and a ROI outside the Eppendorf tubes to determine the average background signal. Images were corrected for physical 
decay to account for signal loss. The radionuclide detection limit was defined as the activity concentration where the Signal-to-Noise Ratio (SNR) is equal to $2 .{ }^{11}$

\subsection{Clinical study}

The currently ongoing clinical feasibility study was approved by the local Medical Ethics Committee (NL8256) and all patients provided written informed consent. Inclusion criteria are sufficient uptake on preoperative PSMA PET/CT-scan (2 times the CLI detection limit) and a primary tumor larger than $1.5 \mathrm{~cm}$ on T2-TSE weighted MRI sequence to ensure sufficient quantitative assessment on PET/CT. Additionally, patients should be scheduled for robot assisted laparoscopic prostatectomy (RALP). The $\mathrm{SUV}_{\text {mean }}$ (Standard Uptake Value) of the benign prostate tissue and tumor were extracted from PSMA PET/CT using OsiriX. A VOI encapsulating the entire prostate was used to estimate the total amount of radioactivity in the prostate, and accordingly, the percentage of the total injected activity was calculated. This percentage injected dose on clinical PET was used to compare the CLI radiances of different patients.

The workflow of the clinical study is visualized in Figure 1. After administering local anesthesia and positioning the da Vinci robot (Intuitive Surgical Inc., Sunnyvale, USA) approximately $100 \mathrm{MBq}{ }^{68} \mathrm{Ga}-\mathrm{PSMA}$ was administered intravenous in the operating room. During the entire procedure the radiation exposure of the operating staff was monitored using electronic personal radiation dosimeters (MGP Instruments DMC 2000; Mirion Technologies, San Ramon, USA). After resection, the prostate specimen was carefully rinsed with $\mathrm{NaCl}$, to remove superficial contamination of radioactive urine and blood. Ex vivo CLI images of the prostate specimen were acquired in the operating room without interfering the surgical procedure. From all sides of the prostate (left, right, anterior, posterior, apex, and base) acquisitions were made with different predefined imaging protocols. Acquisition settings varied the exposure time $(30,60,150,300 \mathrm{~s})$, pixel binning $(2 \times 2[32 \mu \mathrm{m}], 4 \times 4[64 \mu \mathrm{m}], 8 \times 8[128 \mu \mathrm{m}])$ optical filters (no filter, $550 \mathrm{~nm}$ shortpass filter, $800 \mathrm{~nm}$ shortpass filter) and different number of frames $(3,4,5,6)$. In general, an exposure time of 150s consists of 3 frames of 50s. If frame numbers were varied, the total exposure time was kept constant, by changing the number of frames and the duration of that frame. Binning, exposure times, optical filters and kinetic series were only varied in the first four patients to determine the most suitable clinical imaging protocol for all subsequent patients. Suitable in this context, means an acquisition time $<30$ minutes for all 6 sides of the prostate and preferably a tumor to background ratio (TBR) bigger than 2 .

Areas with increased CLI signal (i.e., hotspots) identified during acquisition were marked with a suture to allow direct correspondence with the histopathological analysis, which was performed as standard of care. A PSM was defined as tumorous tissue extending into the inked surface ${ }^{12}$. The bottom $1 \mathrm{~cm}$ of the prostate (i.e., apex) was cleaved and imaged to enable tumor visualization and confirm detection of ${ }^{68} \mathrm{Ga}-\mathrm{PSMA}$ accumulation with CLI.

Postoperative data analysis was performed using MATLAB R2017b (The MathWorks, Natick, USA, 2017), without knowledge of the histopathology results. An empty background image was subtracted from the CL image before analysis. The average radiance (photons $/ \mathrm{s} / \mathrm{cm}^{2} / \mathrm{sr}$ ) was obtained by manually selecting ROIs on each side of the prostate. Radiances obtained from all subsequent images were corrected for radioactive decay (Radiance ${ }_{\text {corr }}$ ). To enable comparison of radiance levels between patients, the radiance was corrected for injected activity. On the cleaved prostate, ROIs were placed over tumorous and benign tissue to determine local radiance levels, and to identify a patient-specific reference value to label specific hotspots as PSM or NSM (Negative Surgical Margin). 


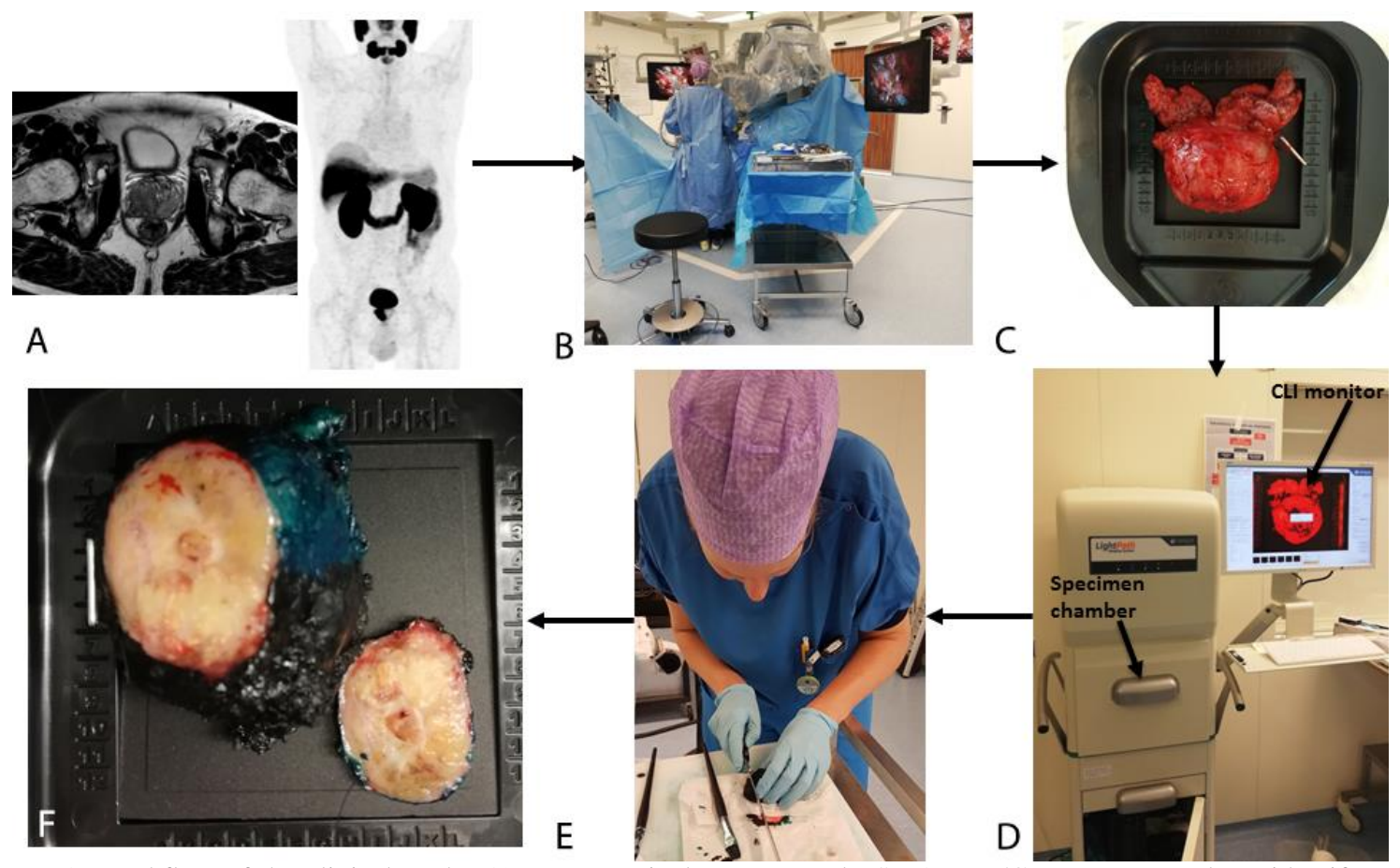

Figure 1. Workflow of the clinical study. A) Pre-operatively a MRI and PSMA PET/CT-scan are made to identify suitable patients for this study and to estimate PSMA uptake in the prostate. B) During the robot assisted laparoscopic prostatectomy ${ }^{68} \mathrm{Ga}$-PSMA is injected intravenously. C) The resected prostate is rinsed with $\mathrm{NaCl}$, placed on the specimen tray, and (D) inserted into the specimen chamber for imaging with the LightPath device. Multiple acquisitions are performed subsequently. E) After imaging the intact specimen and marking the hotspots with a suture, the specimen is inked for orientation. The bottom $1 \mathrm{~cm}$ of the apex is cleaved, to ensure direct tumor visualization. F) The cleaved prostate is positioned on the specimen tray and imaged again.

\section{RESULTS}

\subsection{Gallium-68 validation experiments}

${ }^{68} \mathrm{Ga}$-PSMA uptake in primary PCa on PET/CT ranged between $1.59-8.45 \mathrm{kBq} / \mathrm{mL}$ roughly 45 minutes after tracer injection $(93.7 \pm 8.6 \mathrm{MBq})$. Using CLI with exposure time of 120 s and $2 \times 2$ binning, the minimal detectable concentration for ${ }^{68} \mathrm{Ga}$ was $1.2 \mathrm{kBq} / \mathrm{mL}$ without, and $1.8 \mathrm{kBq} / \mathrm{mL}$ with additional tissue. For ${ }^{18} \mathrm{~F}$ these values were $23.7 \mathrm{kBq} / \mathrm{mL}$ and $39.2 \mathrm{kBq} / \mathrm{mL}$, respectively. Using CLI with E300B8, the minimal detectable concentration for ${ }^{68} \mathrm{Ga}$ was $0.1 \mathrm{kBq} / \mathrm{mL}$ without, and $0.2 \mathrm{kBq} / \mathrm{mL}$ with additional tissue. For ${ }^{18} \mathrm{~F}$ these values were $3.4 \mathrm{kBq} / \mathrm{mL}$ and 5.0 $\mathrm{kBq} / \mathrm{mL}$, respectively. For both isotopes, an excellent linear relationship between the amount of radioactivity and detected light yield was observed $\left(\mathrm{R}^{2}=0.99\right)$, see Figure $2 .{ }^{68} \mathrm{Ga}$ showed approximately $22 \times$ more light yield compared to ${ }^{18} \mathrm{~F}$, thus allowing for shorter acquisition times and/or lower radioactivity concentrations in the clinical setting. The spatial resolution (FWHM) was $1.31 \mathrm{~mm}$ and $1.40 \mathrm{~mm}$ for ${ }^{18} \mathrm{~F}$ and ${ }^{68} \mathrm{Ga}$ respectively without tissue and was $2.4 \mathrm{~mm}$ and $2.73 \mathrm{~mm}$ with tissue using the acquisition setting of exposure time of $120 \mathrm{~s}$ and $2 \times 2$ binning in both conditions. 

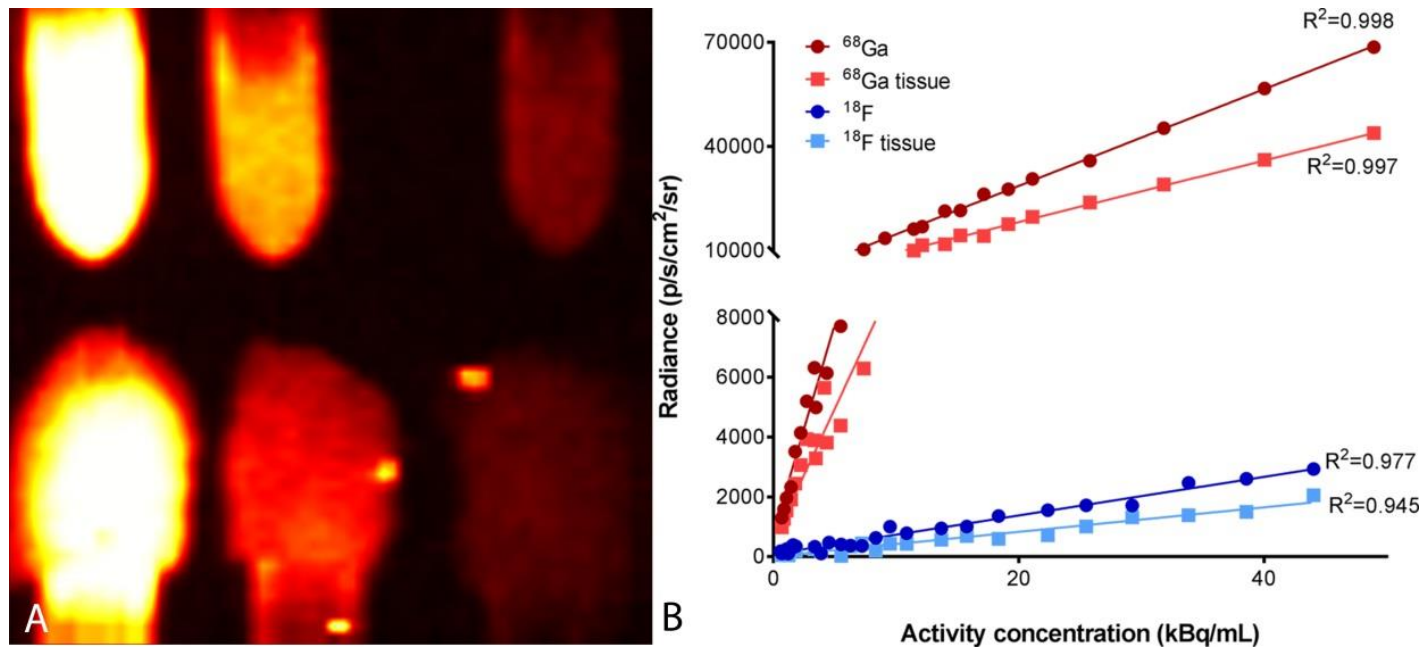

Figure 2: A) A CLI image of 6 Eppendorf tubes filled with ${ }^{68} \mathrm{Ga}$; the bottom row is overlaid with $1 \mathrm{~mm}$ chicken tissue. Activity concentrations are $45,12.5$ and $2.5 \mathrm{kBq} / \mathrm{mL}$ (left to right), in both rows. B) Signal intensity of ${ }^{18} \mathrm{~F}$ and ${ }^{68} \mathrm{Ga}$ with respect to the absolute activity concentration, as well as the effect of tissue on the signal. The goodness-of-fit $\left(\mathrm{R}^{2}\right)$ is displayed at every fit. Data was acquired with an exposure time of 120 s and $2 \times 2$ binning. Note: the intense spots are gamma strikes.

\subsection{Clinical study}

Eight primary prostate cancer patients were enrolled in this ongoing prospective study. The administered radioactivity was between $23-118 \mathrm{MBq}$, and the time between injection and CLI imaging ranged from 59-95 min. The absorbed radiation dose to the personnel during surgery ranged from $0.002-0.016 \mathrm{mSv}$ depending on the position with regard to the patient.

The different acquisition protocols as mentioned in the previous section were tested on four specimens, see Figure 3 . The accompanying TBR can be found in Table 1. The TBR increased when using more pixel binning, a longer exposure time, or without any filtering, as could be expected. However, as timing is important in the operating theater, the increase in TBR at 300s as compared to 150 s (1.98 vs 1.85), was considered minor while doubling the exposure time. Therefore, $150 \mathrm{~s}-8 \times 8$ bin seemed the most appropriate. Next to that, three frames is the minimum in order to reduce the effect of gamma strikes. The absence of optical filtering showed the highest TBR, however, this setting complicates distinguishing signals that originate superficially from deeper ones. As this is an essential feature for margin assessment, an image with a $550 \mathrm{~nm}$ shortpass filter will be acquired if a hotspot is visualized without optical filtering.

Table 1. Overview of different acquisition protocols and the effect on the tumor to background ratio (TBR). E= exposure time (s), $\mathrm{B}=$ pixel binning, $\mathrm{F}=$ Filter $(\mathrm{F} 0=$ no filter, $\mathrm{F} 550=550 \mathrm{~nm}$ shortpass, $\mathrm{F} 800=800 \mathrm{~nm}$ shortpass filter). Fr =frames, if not specified 3 frames were used. Final protocol was E150B8 with F0 and F550, seen as bold in the table.

\begin{tabular}{|c|c|c|c|c|c|}
\hline \multirow{2}{*}{ Exposure time } & Acquisition & E30B8F550 & E60B8F550 & E150B8F550 & E300B8F550 \\
\cline { 2 - 6 } & TBR & 1.10 & 1.18 & 1.85 & 1.98 \\
\hline \multirow{2}{*}{ Frames } & Acquisition & 3Fr×50sB8F0 & 4Fr×37sB8F0 & 5 Fr×30sB8F0 & 6Fr×25sB8F0 \\
\cline { 2 - 6 } & TBR & 4.33 & 3.82 & 3.74 & 3.35 \\
\hline \multirow{2}{*}{ Binning } & Acquisition & E150B8F0 & E150B8F550 & E150B8F800 & \\
\cline { 2 - 6 } & TBR & 4.33 & 1.85 & 3.88 & \\
\cline { 2 - 6 } & Acquisition & E150B2F550 & E150B4F550 & E150B8F550 & \\
\cline { 2 - 6 } & TBR & 1.02 & 1.26 & 1.85 & \\
\hline
\end{tabular}




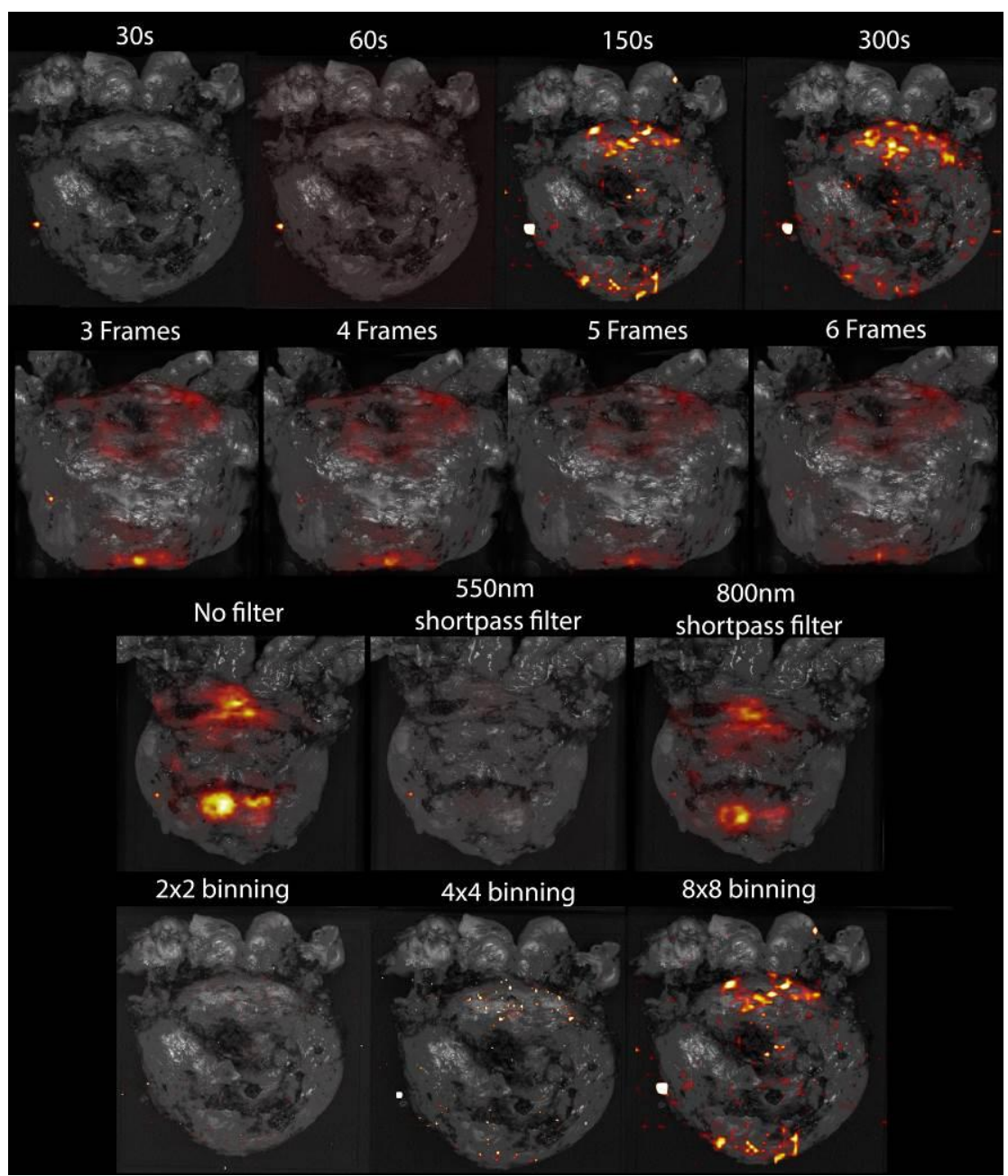

Figure 3. Fused CLI and white light images of the anterior side of the prostate specimen. The first and the last row is the specimen of the same patient. In the first row, the exposure time is varied, images were taken with $8 \times 8$ binning, 3 frames and $550 \mathrm{~nm}$ shortpass filter. The second row the number of frames are varied, with a constant total exposure time $(150 \mathrm{~s}), 8 \times 8$ binning and no optical filter. The third row includes imaging using different filters, acquired with 150 s exposure time, 3 frames and $8 \times 8$ binning. In the last row the binning was varied, with an exposure time of $150 \mathrm{~s}$ and $550 \mathrm{~nm}$ filter.

The bottom $1 \mathrm{~cm}$ of the apex was cleaved in five prostate specimens, to ensure direct visualization of the benign and tumor tissue (see Figure 4). The median radiance ${ }_{\text {corr }}$ of the cleaved tumor on E150B8F550 was $2001 \mathrm{p} / \mathrm{s} / \mathrm{cm}^{2} / \mathrm{sr}$ (range: 1393-2642), compared to $550 \mathrm{p} / \mathrm{s} / \mathrm{cm}^{2} / \mathrm{sr}$ (range: 424-694) of the benign region. On the filtered intact prostate images the median radiance corr $_{\text {of }}$ the benign tissue was $584 \mathrm{p} / \mathrm{s} / \mathrm{cm}^{2} / \mathrm{sr}$ (range: 164-1441) and of a suspicious hotspot the signal was $2491 \mathrm{p} / \mathrm{s} / \mathrm{cm}^{2} / \mathrm{sr}$ (range: 798-9129), see Table 2.

Out of the eight patients, a PSM was actually detected in three patients according to histopathology, and all these proven PSM's corresponded to suspicious locations on CLI acquisitions. In the remaining five patients a NSM was reported according to histopathology, which was in concordance with the CL images in two patients. A true-positive PSM was even detected in a patient where the radioactivity was very low (12MBq), and preoperative PET/CT measurements showed that in this patient roughly $0.4 \%$ of the injected activity accumulates in the prostate (see Figure 5). In three specimens, however, false-positive suspicious regions were detected on CLI. In these three patients additional histopathological assessment showed that the tumor was located within $0.1 \mathrm{~mm}$ from the specimen surface at the marked locations. 


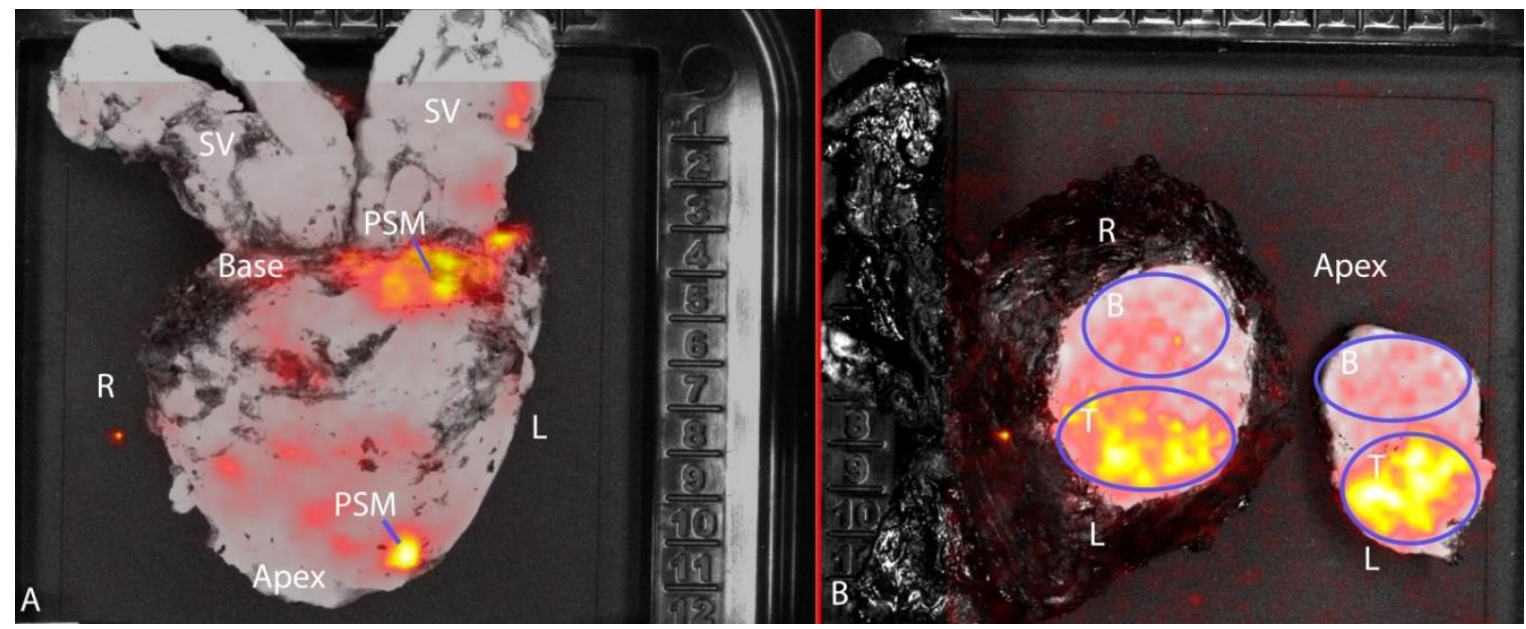

Figure 4. A) A fused CLI image with the white light image of the anterior side of the prostate. The blue lines indicate the confirmed positive surgical margins. B) The cleaved prostate $(1 \mathrm{~cm}$ apex was cleaved), with the fused CLI image. In this specific patient, the tumor was located on the left side of the prostate, confirmed by preoperative ${ }^{68}$ Ga-PSMA PET/CT scan. The ROIs with $\mathrm{T}$ contain tumor and $\mathrm{B}$ benign tissue. $\mathrm{R}$ and $\mathrm{L}$ indicate right and left sides and $\mathrm{SV}$ the seminal vesicles. 


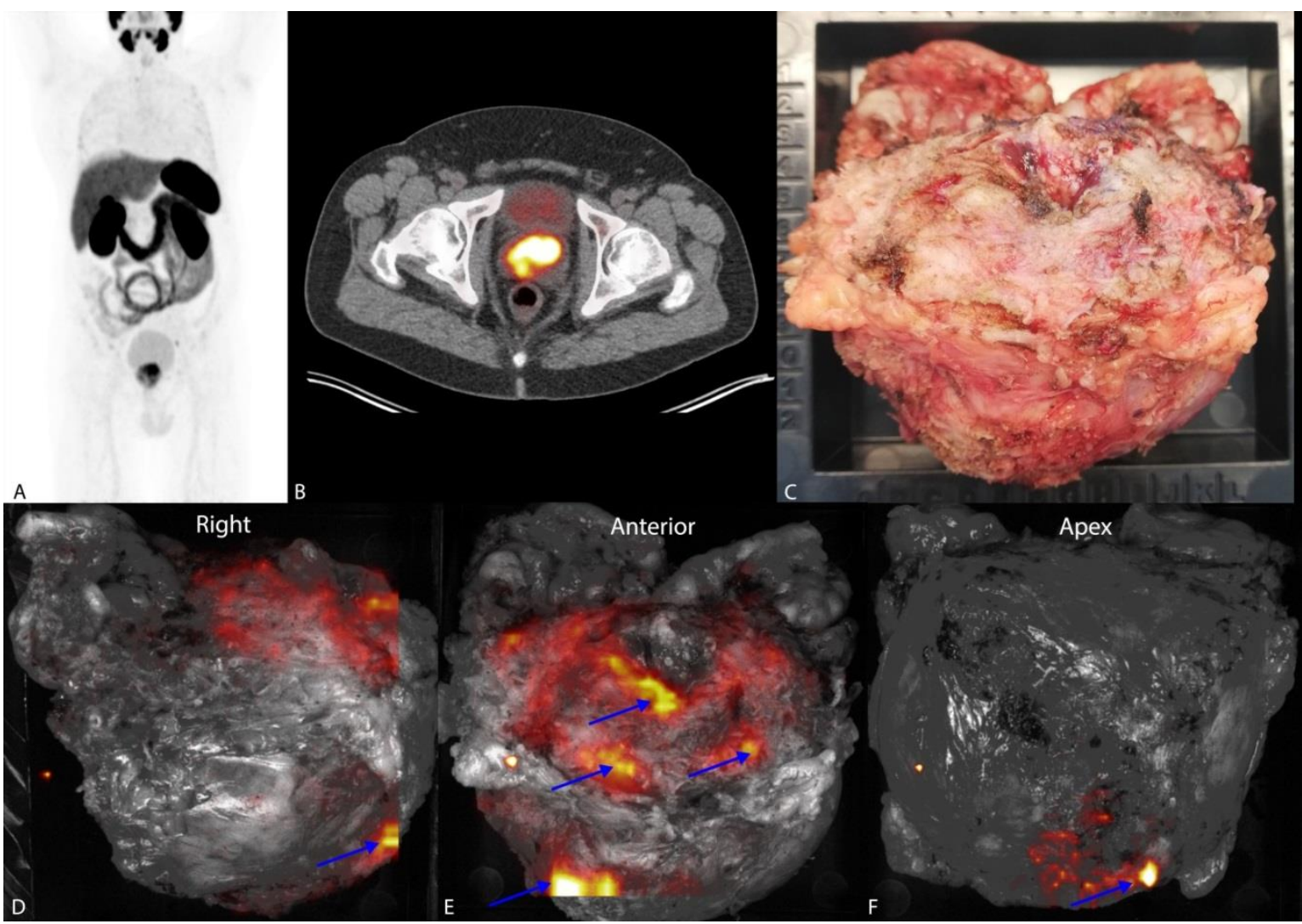

Figure 5: A) A maximum intensity projection of the PSMA PET/CT scan. B) The fused PET/CT scan at height of the prostate tumor, showing clear PSMA uptake in the tumor. C) A white light image of the anterior side of the excised intact prostate. D-F) A fused CLI image with the white light image of the right, anterior side and apical of the prostate, the blue arrows indicate the confirmed positive surgical margin. Note: in this patient only $12 \mathrm{MBq}$ was present in the patient at the time of imaging, of which $0.4 \%$ is expected in the prostate according to the preoperative PET/CT.

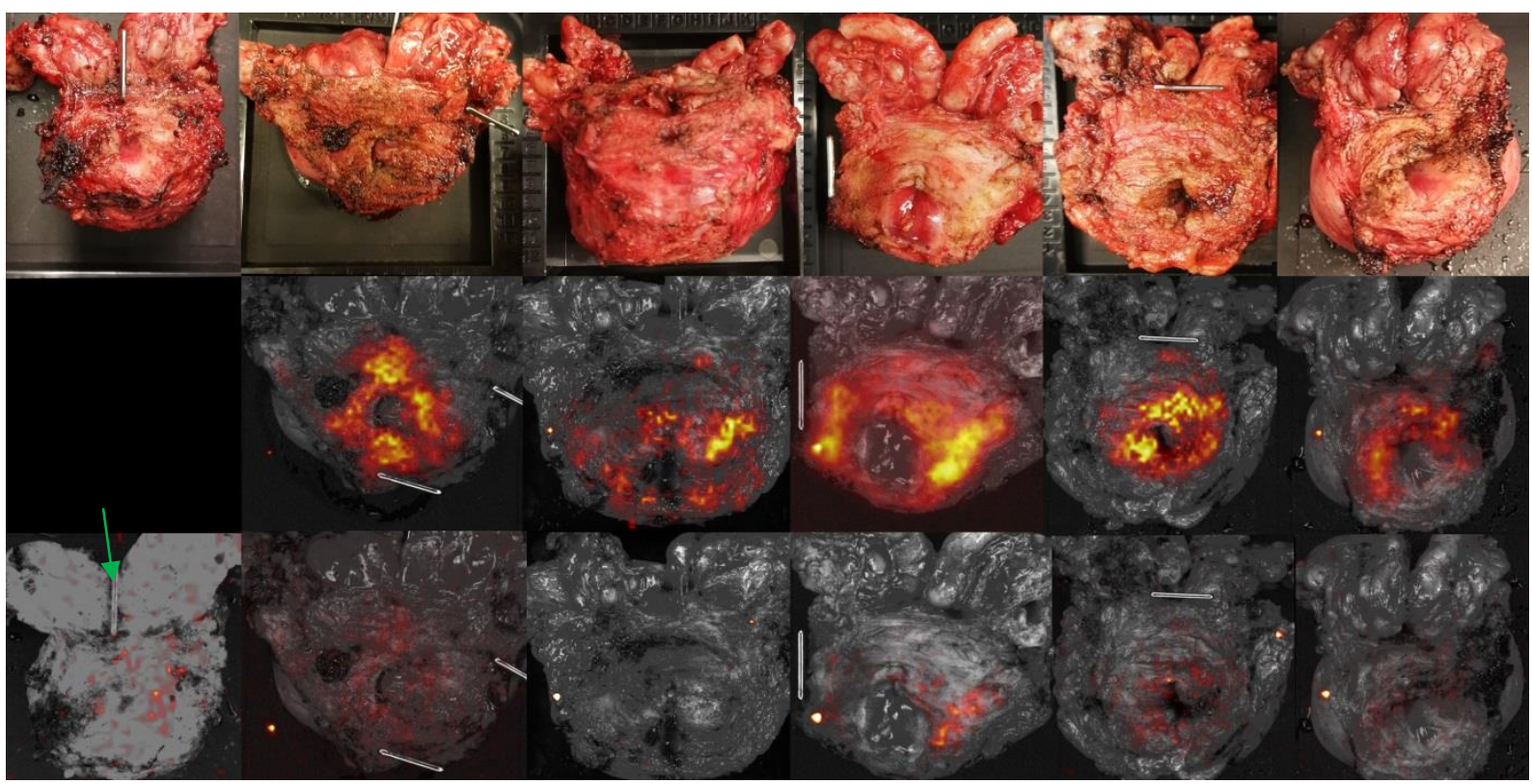

Figure 6. The upper row contains white light images of the base of the prostate of 6 patients. The middle row shows the fused unfiltered CLI image of the base. The bottom row shows the same acquisition as in the center row with a $550 \mathrm{~nm}$ shortpass filter. The patient displayed in the most left column did not have an unfiltered image of the basal side of the prostate. There are gray pins present to position the prostate, see green arrow. 
In six out of eight patients a halo-like structure was observed on the basal side of the prostate (Figure 6). For now, we hypothesize that the halo is caused by contamination of the specimen with radioactive urine, since it is only visible on the basal side which is in close proximity to the bladder. In one patient we tested the urine contamination hypothesis, by additional rinsing to remove urine (see Figure 7). Between the unrinsed and rinsed image there is a clear difference in intensity. However, after rinsing twice and correcting for the decay (Figure 7D), the intensity looks similar to the one in 7B, suggesting that the radioactive urine cannot be rinsed that easily from the specimen's surface.

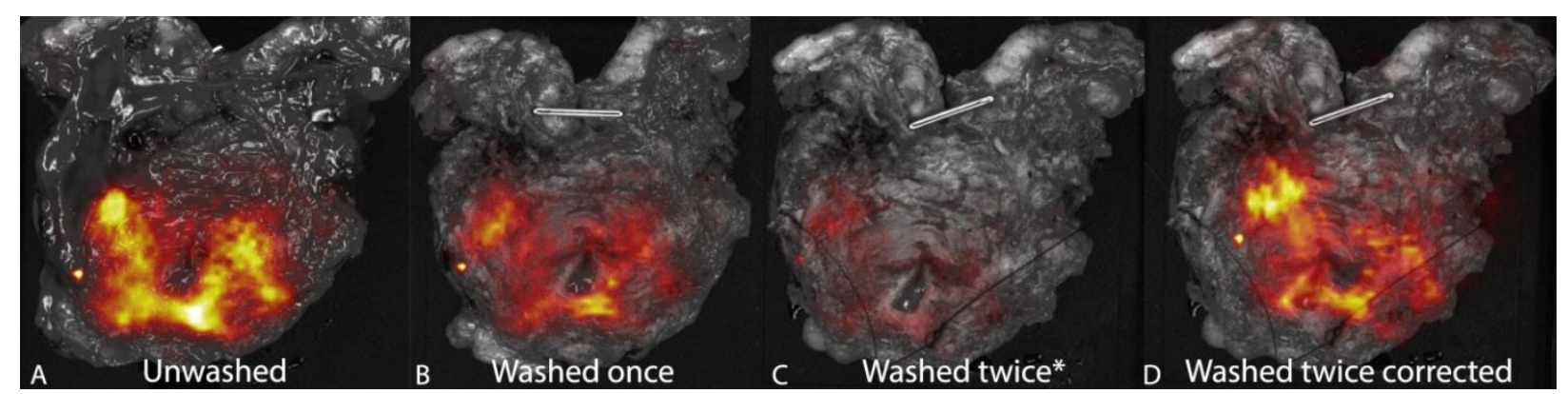

Figure 7: The basal side of the prostate A) unrinsed, B) rinsed once in $\mathrm{NaCl}$ and $\mathrm{C}$ ) rinsed twice in $\mathrm{NaCl}$, however the imaging was performed $55 \mathrm{~min}$ later D) image $\mathrm{C}$, but then corrected for the decay within $55 \mathrm{~min}$. All images are scaled with the same intensity range. 
Table 2. Overview of quantitative analysis of the PET/CT scan and CLI images. The SUV mean of the benign tissue and the tumor tissue were retrieved from the PSMA PET/CT scan, and the corresponding Tumor to Background ratio (TBR). The mean radiance $_{\text {corr }}$ of a hotspot on both the filtered and the unfiltered CLI image, and the corresponding TBR. In five patients the bottom $1 \mathrm{~cm}$ of the apex was cleaved, on which direct tumor measurements could be performed. The mean radiance corr $_{\text {of }}$ the tumor and benign tissue and TBR are displayed here, both for the filtered and unfiltered CLI image. All radiances were retrieved from images with an exposure time of 150s and $8 \times 8$ binning, and were corrected for decay and injected dose. SUV= Standard Uptake Value.

\begin{tabular}{|c|c|c|c|c|c|c|c|c|}
\hline Patient & 1 & 2 & 3 & 4 & 5 & 6 & 7 & 8 \\
\hline $\begin{array}{l}\mathrm{SUV}_{\text {mean }} \\
\text { benign }\end{array}$ & 2.0 & 1.7 & 1.8 & 2.0 & 1.9 & 2.4 & 3.1 & 3.0 \\
\hline $\begin{array}{l}S U V_{\text {mean }} \\
\text { tumor }\end{array}$ & 9.7 & 13.8 & 3.3 & 17.8 & 4.0 & 9.0 & 11.4 & 13.2 \\
\hline TBR PET & 4.8 & 7.9 & 1.8 & 8.9 & 2.2 & 3.8 & 3.6 & 4.4 \\
\hline $\begin{array}{l}\% \text { injected } \\
\text { dose in } \\
\text { prostate on } \\
\text { PET }\end{array}$ & 0.25 & 0.18 & 0.07 & 0.22 & 0.10 & 0.11 & 0.21 & 0.40 \\
\hline $\begin{array}{l}\text { Activity at } \\
\text { start CLI } \\
\text { (MBq) }\end{array}$ & 45 & 51 & 37 & 42 & 52 & 34 & 29 & 12 \\
\hline $\begin{array}{l}\text { Mean radiance } \\
\text { tumor cleaved } \\
\text { prostate } \\
\mathbf{F 5 5 0 n m / F 0} \\
\left(\mathbf{p} / \mathbf{s} / \mathbf{c m}^{2} / \mathbf{s r}\right)\end{array}$ & 2186/- & $\begin{array}{l}1393 / \\
12554\end{array}$ & - & $\begin{array}{l}1783 / \\
16159\end{array}$ & - & $\begin{array}{l}2642 / \\
18430\end{array}$ & - & - \\
\hline $\begin{array}{l}\text { Mean radiance } \\
\text { benign tissue } \\
\text { on cleaved } \\
\text { prostate } \\
\text { F550nm/F0 } \\
\left(\mathrm{p} / \mathrm{s} / \mathrm{cm}^{2} / \mathrm{sr}\right) \\
\end{array}$ & 694/- & $\begin{array}{l}635 / \\
6820\end{array}$ & $-1 / 6661$ & $\begin{array}{l}424 / \\
6206\end{array}$ & - & $\begin{array}{c}447 / \\
10180\end{array}$ & - & - \\
\hline $\begin{array}{l}\text { TBR cleaved } \\
(\mathrm{F550nm} / \mathrm{F0}) \\
\end{array}$ & $3.1 /-$ & $2.2 / 1.8$ & - & $4.2 / 2.6$ & - & $5.9 / 1.8$ & - & - \\
\hline $\begin{array}{l}\text { Mean radiance } \\
\text { hotspot intact } \\
\text { p/s/cm } 2 / \text { sr } \\
(\mathbf{F 5 5 0 n m} / \mathbf{F 0})\end{array}$ & $1479 /-$ & $\begin{array}{l}1446 / \\
16691\end{array}$ & $\begin{array}{c}798 / \\
11139\end{array}$ & $\begin{array}{l}1617 / \\
16474\end{array}$ & $\begin{array}{l}2603 / \\
17159\end{array}$ & $\begin{array}{l}1264 / \\
12500\end{array}$ & $\begin{array}{l}1595 / \\
17189\end{array}$ & $\begin{array}{l}9129 / \\
78988\end{array}$ \\
\hline $\begin{array}{l}\text { TBR intact } \\
\text { (F550nm/F0) }\end{array}$ & $3.3 /-$ & $4.0 / 4.7$ & $2.5 / 2.5$ & $3.3 / 2.7$ & $2.1 / 1.9$ & $7.7 / 2.5$ & $7.8 / 2.3$ & $6.3 / 5.6$ \\
\hline $\begin{array}{l}\text { PSM on } \\
\text { histopathology }\end{array}$ & Yes & Yes & No & No & No & No & No & Yes \\
\hline $\begin{array}{l}\text { Distance } \\
\text { tumor to } \\
\text { surface (mm) }\end{array}$ & 0 & 0 & 3 & 0.1 & 1.5 & 0.1 & 0.01 & 0 \\
\hline
\end{tabular}




\section{DISCUSSION}

The results of the initial ${ }^{68} \mathrm{Ga}$ validation experiments and subsequent transition towards a clinical CLI protocol in a limited number of patients, shows that it is technically feasible to assess the prostate margins with CLI during surgery. The in vitro experiments demonstrated that the spatial resolution of CLI for ${ }^{68} \mathrm{Ga}$ is comparable to ${ }^{18} \mathrm{~F}$. It is also important to note that the experiments showed that radiance level of ${ }^{68} \mathrm{Ga}$ is roughly $22 \times$ higher, making it far more suitable for CLI than ${ }^{18} \mathrm{~F}$. Thus, ${ }^{68} \mathrm{Ga}$-PSMA-CLI should be able to detect clinically relevant radioactivity levels on a specimen after administration of $\sim 100 \mathrm{MBq}{ }^{68} \mathrm{Ga}$-PSMA. However, the optimal acquisition settings for in vitro imaging (E120B2) are not per definition the best choices for clinical ex vivo imaging. Factors that may degrade the signal, such as photon attenuation and scattering in tissue or the presence of radioactive urine, are difficult to mimic in vitro. Therefore, the protocol was optimized in the clinical ex vivo situation.

As with any technology that will be used in the medical domain, there are pure practical requirements to have to be med. For surgical use of an imaging technique these requirements include: 1) low dosage of radioactivity to limit the radiation exposure to OR-staff, 2) acquisition within 30 minutes enabling direct margin-feedback to the surgeon, and 3) precise distinction between tumor-positive and -negative areas to prevent false positive/negative results. These requirements were taken into account during protocol optimization and feasibility evaluation of ${ }^{68} \mathrm{Ga}$-PSMA CLI for intra-operative margin assessment. Eventually, all six sides of the prostate were imaged with an acquisition time of $150 \mathrm{~s}$ ( 3 frames of 50s) and a pixel binning of $8 \times 8$, resulting in a minimal imaging time for the entire specimen of 15 minutes. In the clinical ex vivo situation, the signal is acquired in 3 frames to reduce the influence of gamma strikes. The latter are $511 \mathrm{keV}$ annihilation photons emitted from the radionuclide, visualized as a local high signal spikes with a characteristic tail (Figure 2). Pixel binning of $8 \times 8$ resulted in a slight reduction of noise, while maintaining detailed spatial information.

If a hotspot was identified on the prostate surface, additional imaging with a $550 \mathrm{~nm}$ shortpass filter proved useful for further PSM identification. In the last four patients, first a CL image was made without an optical filter and when hotspots were identified, the acquisition was repeated with a $550 \mathrm{~nm}$ shortpass filter. If the intense signal was still present on the filtered image there is a higher probability of PSMA-containing cells near the surface, since light with short wavelengths originating from deeper cell layers is more likely to be attenuated and scattered in tissue. The isotope ${ }^{68} \mathrm{Ga}$ has an average positron range of $2.8 \mathrm{~mm}^{13}$, so Cerenkov photons can be produced along this entire path, and accordingly, it remains difficult to estimate the exact depth at which the signal originates with just one optical filter. Calvert et al. performed a simulation study to estimate the penetration depth of the Cerenkov signal using ${ }^{18} \mathrm{~F}$ ${ }^{14}$. However, these results cannot be converted as ${ }^{68} \mathrm{Ga}$ has a different positron range than ${ }^{18} \mathrm{~F}$ (average range 0.5 $\mathrm{mm}^{13}$ ). The latter phenomena can well be an explanation for the false positive margins using CLI found in this study. In all three patients, false-positive PSMs were found in close proximity to the specimen surface $(\leq 0.1 \mathrm{~mm})$.

While the definition of an adequate surgical margin on histopathology varies over time and is always subject of debate, the current histopathological definition in PCa of PSM is 'tumor on ink' or ' $0 \mathrm{~mm}$ from the surface'. When adhering to this definition it seems ambitious to use ${ }^{68} \mathrm{Ga}$-PSMA-CLI to guide the surgical procedure, as the Cerenkov-signal may arise from depth up to $2.8 \mathrm{~mm}^{13}$. Still, we believe that CLI can provide valuable information on margin-status, as it may guide surgeons to areas at risk so possibly a wider resection could be performed on that location. Additionally, marking regions at risk may also be beneficial for the pathologist as more precise histopathological evaluation is then possible.

In the first patients that were included in the feasibility study, there was permission granted from the pathologist to cleave the lower part of the prostate specimen. This enabled direct measurements of the radiance corr $_{\text {in tumorous and }}$ benign tissue. The average intensity of the cleaved tumor $\left(2001 \mathrm{p} / \mathrm{s} / \mathrm{cm}^{2} / \mathrm{sr}\right)$ was quite comparable to the average radiance $_{\text {corr }}$ of the identified hotspots $\left(2491 \mathrm{p} / \mathrm{s} / \mathrm{cm}^{2} / \mathrm{sr}\right)$. It is important to note that the hotspot-ROI is generally much smaller than the cleaved tumor-ROI, and therefore, the average radiance corr $_{\text {in }}$ the hotspot is slightly higher. The radiance $_{\text {corr }}$ in benign tissue on the cleaved and intact prostate was comparable as well $\left(550 \mathrm{vs} 584 \mathrm{p} / \mathrm{s} / \mathrm{cm}^{2} / \mathrm{sr}\right.$ respectively). Based on these measures we want to propose a methodology to discriminate between benign and tumor based on quantitative values, rather than on visual interpretation alone in a forthcoming paper. 
Visual assessment is inherently prone to incorrect window-level settings, which may result in erroneous identification of hotspots. The process of cleaving is not preferred in the eventual work up, as it is time intensive and requires additional training.

There is one important drawback of CLI, or any technique that involves the usage of radioactivity during OR, and that is the radiation dose to especially OR-personnel. In the current study, ${ }^{68} \mathrm{Ga}$ administrations were only performed by skilled workers from the nuclear medicine department, and exposure to the OR-personnel was closely monitored. The highest radiation dose was $0.0016 \mathrm{mSv}$ per surgery, measured on the scrub nurse located directly next to the patient. This number was two times lower than a comparable study performed with ${ }^{18}$ F-FDG-CLI in breast cancer ${ }^{6}$. This specific study administered $\sim 295 \mathrm{MBq}$ 18F-FDG for assessment of breast margins, and acquired images at 300 with $8 \times 8$ binning.

\section{Study limitations}

There was a large range in the injected activity (23-118 MBq). Thus, a head to head visual comparison is difficult considering the influence of activity on signal intensity. Still, all radiances were corrected for the injected activity, due to the linear relationship between radiance and activity, as investigated in the in vitro study.

The short half-life (68 min) of ${ }^{68} \mathrm{Ga}$-PSMA is a disadvantage for PSM detection using CLI, since surgeries have a tendency to start later than planned, resulting in a large variation of injected activity, this cannot be compensated easily. Nonetheless, even with very low amounts of injected activity (i.e. $23 \mathrm{MBq}$ ); CLI images were still appropriate for quantitative and qualitative assessment to detect a PSM (Figure 5). Next, as can be seen in Figure 5, the prostate should not be too large, since parts of this prostate $(70 \mathrm{cc})$ fell outside the Field of View.

The basal halo hampers the visual and quantitative assessment of the prostate base. It has not been described in literature that there is more PSMA expression at the base of the prostate. Thus unlikely that the halo is induced by benign PSMA expression. For now, we hypothesize that the halo is caused by contamination of the specimen with radioactive urine, since it is only visible on the basal side which is in close proximity to the bladder. This hypothesis need further exploration.

The used inclusion criteria induced a bias for the number of PSMs. As PSMA PET/CT-scans are only made for high risk prostate cancer patients and the number of PSM is higher in a higher grade. Despite this fact, this target group might benefit the most of the usability of CLI. As low grade prostate PCa occurs smaller, organ confined lesions, have a lower chance on PSM.

\section{CONCLUSION}

${ }^{68} \mathrm{Ga}$ is superior over ${ }^{18} \mathrm{~F}$ in terms of light yield and detection limit, yet the resolution was worse for ${ }^{68} \mathrm{Ga}$. CLI proved sensitive enough to detect the average ${ }^{68} \mathrm{Ga}$ uptake measured on clinical PET images. The standard clinical activity $\sim 100 \mathrm{MBq}$ is sufficient for intraoperative use with a suitable CLI-imaging protocol, meaning a short acquisition time and clinically relevant sensitivity.

Preliminary results showed that CLI was able to detect PSM ex vivo, with a protocol which can assess all sides of the prostate within 15 minutes. In some cases, CLI showed a suspicious area for a PSM; however, histopathology indicated that the tumor was $0.1 \mathrm{~mm}$ from the edge. Based on these preliminary results CLI holds promise for intraoperative margin assessment in PCa, the ongoing trial and future work will evaluate the diagnostic accuracy of CLI in a larger population of PCa patients.

\section{ACKNOWLEDGMENTS}

This research is supported by KWF Kankerbestrijding and Technology Foundation STW (Grant number 15175). 


\section{REFERENCES}

[1] Yossepowitch, O., Briganti, A., Eastham, J.A., Epstein, J., Graefen, M., Montironi, R., and Touijer, K., "Positive Surgical Margins After Radical Prostatectomy: A Systematic Review and Contemporary Update," European Urology 65(2), 303-313 (2014).

[2] Ciarrocchi, E., and Belcari, N., "Cerenkov luminescence imaging: physics principles and potential applications in biomedical sciences," EJNMMI Physics 4(1), 14 (2017).

[3] Dothager, R.S., Goiffon, R.J., Jackson, E., Harpstrite, S., and Piwnica-Worms, D., "Cerenkov radiation energy transfer (CRET) imaging: A novel method for optical imaging of PET isotopes in biological systems," PLoS ONE 5(10), 1-7 (2010).

[4] Zhang, R., Fox, C.J., Glaser, A.K., Gladstone, D.J., and Pogue, B.W., "Superficial dosimetry imaging of Čerenkov emission in electron beam radiotherapy of phantoms," Physics in Medicine and Biology 58(16), 5477-5493 (2013).

[5] Tanha, K., Pashazadeh, A.M., and Pogue, B.W., "Review of biomedical Čerenkov luminescence imaging applications," Biomedical Optics Express 6(8), 3053 (2015).

[6] Grootendorst, M.R., Cariati, M., Pinder, S.E., Kothari, A., Douek, M., Kovacs, T., Hamed, H., Pawa, A., Nimmo, F., et al., "Intraoperative Assessment of Tumor Resection Margins in Breast-Conserving Surgery Using 18 F-FDG Cerenkov Luminescence Imaging: A First-in-Human Feasibility Study,” Journal of Nuclear Medicine 58(6), 891-898 (2017).

[7] Corfield, J., Perera, M., Bolton, D., and Lawrentschuk, N., "68Ga-prostate specific membrane antigen (PSMA) positron emission tomography (PET) for primary staging of high-risk prostate cancer: a systematic review," World Journal of Urology 36(4), 519-527 (2018).

[8] Gill, R.K., Mitchell, G.S., and Cherry, S.R., "Computed Cerenkov luminescence yields for radionuclides used in biology and medicine," Physics in Medicine and Biology 60(11), 4263-4280 (2015).

[9] Hu, Z., Wang, W., Gualtieri, E.E., Hsieh, Y.L., Karp, J.S., Matej, S., Parma, M.J., Tung, C.H., Walsh, E.S., et al., "An LOR-based fully-3D PET image reconstruction using a blob-basis function," in 2007 IEEE Nucl. Sci. Symp. Conf. Rec. 6, 4415-4418 (2007).

[10] olde Heuvel, J., de Wit-van der Veen, B.J., Vyas, K.N., Tuch, D.S., Grootendorst, M.R., Stokkel, M.P.M., and Slump, C.H., "Performance evaluation of Cerenkov luminescence imaging: a comparison of 68Ga with 18F," EJNMMI Physics 6(1), 17 (2019).

[11] Hobbie, R.K., and Roth, B.J., [Intermediate physics for medicine and biology] , Springer Science \& Business Media (2007).

[12] Tan, P.H., Cheng, L., Srigley, J.R., Griffiths, D., Humphrey, P.A., Van Der Kwast, T.H., Montironi, R., Wheeler, T.M., Delahunt, B., et al., "International Society of Urological Pathology (ISUP) consensus conference on handling and staging of radical prostatectomy specimens. Working group 5: surgical margins," Modern Pathology 24(1), 48 (2011).

[13] Moses, W.W., "Fundamental limits of spatial resolution in PET," Nuclear Instruments and Methods in Physics Research Section A: Accelerators, Spectrometers, Detectors and Associated Equipment 648(9), S236-S240 (2011).

[14] Calvert, N., Helo, Y., Mertzanidou, T., Tuch, D.S., Arridge, S.R., and Stoyanov, D., "A simulation study of spectral Čerenkov luminescence imaging for tumour margin estimation,” 13 March 2017, Proc. SPIE 10137. 